\title{
Discovery of mcr-1 Harboring Incl2 Plasmids from Clinical Isolates of Multiclonal E. Coli prevalent in Pakistan.
}

Hazrat Bilal

Anhui University

Tayyab ur Rehman

Khyber Medical University

Muhammad Asif Khan

Khyber Medical University

Fareeha Hameed

Khyber Medical University

Zhang gao Jian

Anhui University

Jianxiong Han

Anhui University

Xingyuan Yang ( $\nabla$ xingyuan@ahu.edu.cn )

Anhui University

Research

Keywords: colistin resistance, mcr-1, Incl2, E. coli, Pakistan

Posted Date: September 18th, 2020

DOI: https://doi.org/10.21203/rs.3.rs-74913/v1

License: (1) This work is licensed under a Creative Commons Attribution 4.0 International License. Read Full License 


\section{Abstract}

Background: Colistin is the last resort antibiotic against multiple drug-resistant (MDR) bacteria found in clinical infections; however, the emergence of plasmid-mediated mcr-1 gene annulled the efficacy of Colistin. This study was planned to determine the prevalence of mcr genes in clinical isolates collected in Pakistan. The molecular types and plasmids of $m c r-1$ bearing isolates were analysed.

Methods: A total of 545 E. coli isolates collected from two major cities of Pakistan were screened for colistin-resistance and mcr genes from June 2018 to September 2019. All positive strains were subjected to antimicrobial susceptibility testing, ESBL, and MBL detection via DDST and CDT. ESBL genes detection, molecular typing, conjugation experiment, plasmid replicon typing, S1 PFGE, and southern hybridization were performed.

Results: Four $(0.73 \%)$ strains of mcr-1 positive isolates were susceptible to meropenem, fosfomycin, and chloramphenicol, including one that showed moderate level resistance to chloramphenicol and fosfomycin. All four strains were ESBL positive and harbored the blaCTX-M-15 gene, while three of the isolates also harbored the blaTEM-1 gene. Molecular typing revealed that four isolates belonged to diverse clonal types, mostly (75\%) from avian pathogenic $E$. coli lineage. The $m c r-1$ gene was present on $60 \mathrm{~kb}$ Incl2 plasmid, which was successfully transconjugated.

Conclusion: The mcr-1 gene's detection in diverse clonal types from MDR clinical isolates enforces priority basis large scale surveillance studies followed by corrective actions to prevent the spread of the mcr- 1 gene in clinical, poultry, and environmental setting.

\section{Background}

Antibiotic resistance is a serious concern worldwide. The situation is escalating due to the misuse and overuse of antibiotics. Different antibiotic resistance mechanisms are developed by bacteria, among which the ESBL and MBL production are the most prominent. Most of the MDR bacteria show resistance to more than one drug [1]. Colistin, a cationic polypeptide antibiotic, was considered the last drug of choice against these superbugs [2]. Various chromosomal mediated colistin resistance such as the two-component system pmrAB, phoPQ, and regulator mgrB have to be known in Enterobacteriaceae that alter the lipopolysaccharide (LPS) of the bacterial cell wall, thus losing its affinity of attachment toward polymyxins [3]. However, it was not as high threatening due to their mode of vertical transmission [4]. In November 2015, Liu et al. for the first time discovered a plasmid-mediated mobilized colistin resistance gene from E. coli and Klebsiella Pneumoniae. The gene product belongs to phosphoethanolamine (PEtN) transferases enzymes that add PEtN to the phosphate group of lipid $\mathrm{A}$. This results in the loss of Colistin binding ability to LPS as lipid A more cationic [5]. Later, the colistin resistance $m c r-1$ gene was discovered in 47 countries [6]. It was initially discovered on Incl2 type of plasmid, but later on, it was found on other plasmid incompatibility types from different bacterial isolates collected from humans, animals, food, environment, insect, and water. Besides, other variants of mcr such as $m c r-2, m c r-3, m c r-4, m c r-5, m c r-6, m c r-7, m c r-8$, and $m c r-9$ have been reported from different regions of the world [7].

In Pakistan, mcr-1 harboring E. coli was detected in migratory birds and later on isolated from clinical samples and broiler in Faisalabad City of the Punjab Province of Pakistan [8-12]. In this study, we look for mcr genes ( $m c r-1$ to $m c r-5)$ in 545 E. coli isolates from human samples collected in two major cities Islamabad and Peshawar of Pakistan. We have determined the Antibiotic resistance profile, ESBL genes, ST, plasmid Incompatibility type, and genetic context of $m c r-1$ harboring isolates.

\section{Methods}

\section{Sample collection, isolation, and species confirmation:}

The present study was initiated with the screening of $545 \mathrm{E}$. coli isolates for colistin resistance. All isolates were collected from Microbiology laboratories of PIMS Islamabad $(n=260), \operatorname{HMC}(n=105), \operatorname{RMI}(n=94)$, and KTH $(n=86)$ Peshawar - tertiary care hospitals in Pakistan from June 2018 to September 2019. The isolates obtained from human clinical samples included urine ( $\mathrm{n}=$ $345)$, blood $(n=109)$, stool $(n=18)$, and pus $(n=73)$. The growth capacity of isolates was initially assessed on CLED and 
MacConkey agar. A single colony from each Petri plate was stored in LB media for maintaining strains. Species confirmation of $E$. coli was performed with 16S rDNA PCR using specific primer mentioned in Table S1. The PCR products were sequenced, and species confirmation was performed using EZ Biocloud online software (https://www.ezbiocloud.net/identify).

\section{Phenotypic and molecular detection of colistin resistance:}

Phenotypic detection of colistin resistance was performed applying the broth microdilution method. The Minimum inhibitory concentration results were interpreted according to CLSI guidelines [13]. The genomic DNA from resistant isolates was extracted with the conventional boiling method [14]. The DNA samples were subjected to PCR using mor genes-specific primers mentioned in Table S1. The expected size amplicons were visualized on $1 \%$ Agarose gel, which was sequenced and analysed with BLAST (https://blast.ncbi.nlm.nih.gov/Blast.cgi)

\section{Antimicrobial susceptibility testing:}

The $m c r-1$ harboring isolates were subjected to antimicrobial susceptibility testing using the broth microdilution method. The antibiotics used were Ampicillin, Cefotaxime, Chloramphenicol, Ciprofloxacin, Fosfomycin, Cefoxitin, Gentamycin, Aztreonam, Amikacin, Meropenem, and Tetracycline. ESBL and MBL activity of resistant isolates were also determined by the Double Desk Synergy Test (DDST) and combined desk test (CDT), respectively. The results were interpreted according to CLSI guidelines [13].

\section{PCR detection of ESBL genes:}

ESBL genes blaTEM, blaSHV, blaCTX-M, and blaOXA variants were amplified from the genomic DNA of colistin-resistant isolates with ESBL genes-specific primers mentioned in Table S1. The expected sizes were visualized on $1 \%$ Agarose gel. For further confirmation, PCR products of ESBL genes were sequenced and blast via the NCBI blast tool (https://blast.ncbi.nlm.nih.gov/Blast.cgi).

\section{Molecular typing:}

The multilocus sequence typing of four $m c r-1$ harboring isolates was carried out. The sequence result of 7 alleles, adk, fumC, gyrB, icd, $m d h$, purA, and recA, were determined from the MLST database (http://enterobase.warwick.ac.uk/species/index/ecoli). To further determine the genetic link among mcr-1 harboring isolates, the Xba I PFGE was performed according to PulseNet PFGE protocol [15]. DNA fingerprint analysis and sketch drawing were performed by BioNumerics v.8.0 (Applied Maths, Sint-MartensLatem, Belgium). Clusters were examined by the unweight pair-group method with arithmetic mean (UPGMA) analysis. The Dice similarity coefficient was calculated with a position tolerance of $1.5 \%$, and a dendrogram was created based on the UPGMA.

\section{Transconjugation and PCR based replicon typing:}

In order to detect the transferability of four colistin-resistant isolates, trans-conjugation experiments were performed. These isolates were selected as the donor, and $E$. coli $\mathrm{EC} 600\left(\mathrm{Nal}^{\mathrm{R}}, \mathrm{Rif}^{\mathrm{R}}\right)$ were taken as recipients. The experiment was performed, as described previously [16]. The transconjugants were analysed using antibiotic susceptibility testing and $m c r-1$ specific PCR, as described earlier. The conjugation transfer rates were calculated by dividing the number of transconjugants by the number of donors.

To further investigate the plasmid incompatibility type responsible for $m c r-1$, the plasmid DNA of transconjugants was extracted by the alkaline lysis method [17]. The plasmid type was determined by using the PBRT 2.0 kit (MBK0078, Diatheva, Italy). The PCR products of eight multiplex PCR were visualized on $2 \%$ Agarose gel, and the results were analysed according to the manufactured instructions. 


\section{S1 PFGE and southern blotting:}

S1 PFGE and southern hybridization of the successfully trans-conjugant E. coli EC600 were performed to determine the plasmid location. For S1 PFGE, the bacterial isolates were embedded into $1 \%$ low melting agarose plugs. Following cell lysis and washing, the plugs were pre-incubated in $200 \mu \mathrm{l}$ of $1 \times \mathrm{S} 1$ buffer at $37^{\circ} \mathrm{C}$ for 30 minutes. S1 digestion was performed by ten units of S1 enzymes (Thermo scientific) in $200 \mu$ of $1 x$ S1 buffer for $10 \mathrm{~min}$ at $37^{\circ} \mathrm{C}$. The digestion was stopped by removing enzymes and adding $200 \mu \mathrm{l}$ of $0.5 \mathrm{M}$ EDTA and left for $10 \mathrm{~min}$ at room temperature. Before running on the gel, the plug was incubated in $200 \mu \mathrm{l}$ of TE buffer for 30 minutes. The products were electrophoresed on the CHEF mapper PFGE system (Bio-Rad USA) for $21 \mathrm{~h}$ at 6 $\mathrm{V} / \mathrm{cm}$ with initial switch time $2.5 \mathrm{sec}$ and final switch time $60 \mathrm{sec}$. The plasmids from the gel were transferred onto the nylon membrane via the capillary transfer technique [18]. Southern hybridization of plasmid DNA with a digoxin-labelled mcr-7-specific probe was performed according to kit instructions (Roche Diagnostics, Mannheim, Germany).

\section{PCR mapping of mcr-1 genetic context:}

To explore the genetic context of $m c r-1$, whether pHNSHP45[5] like key genetic component surrounds it, we designed seven pairs of primers targeting ParA, nikB, tnpA, mcr-1, hp, pilN, and vird4 genes. The plasmid DNA for PCR mapping was extracted by the alkaline lysis method and quantified by one drop [17]. The primers used for mapping are mentioned in Table S1. The PCR products were visualized on $1 \%$ Agarose gel and subsequently sequenced and blasted via the NCBI blast tool.

\section{Results}

\section{Bacterial isolation and colistin resistance screening:}

Among the 545 E. coli isolates, four isolates (PKE051, PKE141, PKE196, and PKE211) showed resistance to Colistin on the broth microdilution method. Upon molecular confirmation through PCR, all the four isolates harbored $m c r-1$ genes, while the other $m c r$ genes were absent. Data about the isolates, location, patients, demography, samples, source, and colistin MIC values are presented in Table 1.

Table 1

samples sources, patient demography, and colistin MIC values.

\begin{tabular}{|c|c|c|c|c|c|c|}
\hline \multirow[t]{2}{*}{ Isolates ID } & \multirow[t]{2}{*}{ Microbiology Laboratory } & \multicolumn{2}{|l|}{ patient } & \multirow[t]{2}{*}{ Sample source } & \multirow[t]{2}{*}{ Colistin MIC } & \multirow[t]{2}{*}{ mcr-1 } \\
\hline & & gender & Age & & & \\
\hline PKE051 & PIMS Islamabad & female & $41 y$ & urine & $8 \mathrm{mg} / \mathrm{l}$ & +ve \\
\hline PKE141 & PIMS Islamabad & female & $32 y$ & urine & $16 \mathrm{mg} / \mathrm{l}$ & $+v e$ \\
\hline PKE196 & HMC Peshawar & female & $25 y$ & urine & $4 \mathrm{mg} / \mathrm{l}$ & $+\mathrm{ve}$ \\
\hline PKE211 & RMI Peshawar & male & $28 y$ & pus & $4 \mathrm{mg} / \mathrm{l}$ & $+\mathrm{ve}$ \\
\hline
\end{tabular}

\section{Antibiotic susceptibility testing and resistant genes:}

All colistin-resistant isolates were MDR but were susceptible to meropenem and were MBL negative. Three isolates showed susceptibility to Chloramphenicol, Ciprofloxacin, and Fosfomycin, while one among them was susceptible to Tetracycline. All of the isolates were ESBL positive via DDST. The blaCTXM-15 gene was found in all of the isolates, while three isolates had the blaTEM-1 gene. The complete antibiogram data are presented in Table 2. 
Table 2

Antibiogram of mcr-1 positive Isolates.

\begin{tabular}{|c|c|c|c|c|c|c|c|c|c|c|c|c|c|c|}
\hline \multirow{2}{*}{$\begin{array}{l}\text { Isolates } \\
\text { ID }\end{array}$} & \multicolumn{11}{|c|}{ MIC of Antibiotics in $\mathrm{mg} / \mathrm{L}$} & \multirow{2}{*}{$\begin{array}{l}\text { ESBL } \\
\text { DDST }\end{array}$} & \multirow{2}{*}{$\begin{array}{l}\text { ESBL } \\
\text { genes }\end{array}$} & \multirow{2}{*}{$\begin{array}{l}\text { MBL } \\
\text { CDT }\end{array}$} \\
\hline & AMP & CTX & CAP & CIP & FOX & FOM & GEN & AZT & MEM & AN & TET & & & \\
\hline PKE051 & $>256$ & 128 & 1 & 0.064 & 64 & 8 & 32 & 256 & 0.032 & 256 & 128 & $+v e$ & $\begin{array}{l}\text { CTXM- } \\
15 \text { TEM- } \\
1\end{array}$ & -ve \\
\hline PKE141 & 256 & 64 & 2 & 0.032 & 32 & 4 & 16 & 128 & 0.032 & 128 & 256 & $+v e$ & $\begin{array}{l}\text { CTXM- } \\
15\end{array}$ & -ve \\
\hline PKE196 & $>256$ & 64 & 2 & 1 & 32 & 128 & 64 & 128 & 0.032 & 128 & 128 & $+v e$ & $\begin{array}{l}\text { CTXM- } \\
15 \text { TEM- } \\
1\end{array}$ & -ve \\
\hline PKE211 & 256 & 256 & 8 & 4 & 16 & 32 & 4 & 128 & 0.064 & 64 & 1 & +ve & $\begin{array}{l}\text { CTXM- } \\
15 / \text { TEM- } \\
1\end{array}$ & -ve \\
\hline
\end{tabular}

\section{Molecular typing:}

Our four $m c r-1$ positive isolates were assigned to ST $405(n=1)$, ST $156(n=2)$ and ST $117(n=1)$ according to Warwick MLST Database (http://enterobase.warwick.ac.uk/species/ecoli/allele_st_search). The results sre presented in Table S2. The Xba I PFGE patterns of four mcr-1 positive isolates are shown in figure 2. The PFGE pattern of two isolates from ST 156 shows resemblance to each other while the two other isolates from ST 405 and ST 117 show a different pattern.

\section{Transconjugation and PBRT:}

Assuming that the $m c r-1$ gene is present on the plasmid, a transconjugation experiment was performed. All of the strains were successfully conjugated with E. coli $\mathrm{EC} 600$. The conjugation rates are presented in Table 3. Single colony from the selected plates were analysed for antimicrobial susceptibility testing and resistant genes. The transconjugants showed resistance to Colistin, and the presence of the mcr-1 gene was confirmed via PCR, while the ESBL genes were not detected in transconjugants. To further determine the Incompatibility type of $m c r-1$ harboring plasmid, the PBRT was performed from the transconjugants. The results confirmed that all of the four isolates had Incl 2 type of plasmid, as shown in figure 3.

Table 3

Conjugation rate of mcr-1 positive isolates to E. coli EC600.

\begin{tabular}{|c|c|c|c|c|}
\hline Isolate ID & Transferability & Trans conjugants & Donor & Conjugation rate \\
\hline PKE051 & Yes & $2.1 \times 10 \rrbracket$ & $4.5 \times 10^{2}$ & $2.1 \times 10^{-} \nabla$ \\
\hline PKE141 & Yes & $1.8 \times 10 \rrbracket$ & $7.8 \times 10^{3}$ & $4.3 \times 10^{-4}$ \\
\hline PKE196 & Yes & $2.3 \times 10 \rrbracket$ & $3.9 \times 10^{2}$ & $1.6 \times 10^{-4}$ \\
\hline PKE211 & Yes & $2.7 \times 10 \rrbracket$ & $4.3 \times 10^{2}$ & $1.5 \times 10^{-4}$ \\
\hline
\end{tabular}

\section{S1 PFGE and southern blotting:}


The S1 PFGE results of transconjugants indicate the transfer of a plasmid of size near to $60 \mathrm{~kb}$ from donor strains. The presence of the mcr-1 gene on the plasmid was confirmed from Southern hybridization, as shown in figure 4.

\section{PCR mapping of $m c r-1$ context:}

In all colistin-resistant isolates, six genes in the nearby region of $m c r-1$ were amplified and sequenced. However, the tnpA gene, in terms of amplicon size, showed an unexpected result. The uncertainty was cleared using the other pair of primers for tnpA loci (absence of $I s A p / 1$ ). This time the expected bands were visualized, and the sequencing result showed missing IsAp/1. The genetic contexts of four $m c r-1$ isolates in comparison with pHNSHP45 are shown in figure 5.

\section{Discussion}

This study identified, for the first time, mcr-1 harboring E. coli from Khyber Pakhtunkhwa province and the capital city of Islamabad, Pakistan. The prevalence of mcr- 1 was $0.73 \%$, while three out of four isolates were found in urine samples. To date, only a single case of $m c r-1$ harboring $E$. coli out of $29 \mathrm{ESBL}$ positive clinical isolates from the Punjab province of Pakistan has been reported [11]. In another study, the $m c r-1$ gene in E. coli was reported from migratory birds and broiler in the Faisalabad region [9 12]. The mcr-1 gene has also been found in Klebsiella pneumoniae, Acinetobacter baumannii, and Pseudomonas aeruginosa isolated from clinical samples [19 20].

The four isolates we report here were MDR and ESBL positive and have the blaCTXM-15 gene, while three of them also harbor the blaTEM-1 gene. The co-existence of $m c r-1$ with ESBL has been frequently reported [21-23]. A recent study suggests that since 1980, there is an evolutionary link between ESBL and $m c r-1$; however, this statement needs further confirmation by tracing the mcr-1 gene in archived ESBL isolates, which might provide clues regarding the kinetics over time between ESBL and $m c r-7[24]$. One study suggests that the co-occurrence of mcr-1 and blaCTXM-15 is perhaps due to intricate genetic actions taken under antibiotic pressure [25].

In the present study, the clonal assortment of E. coli is diverse, that is, two of the isolates belong to ST 156, while two belonged to ST405 and ST 117, altogether different sequence types. The diversity in ST proposes that the distribution of plasmid mediated colistin resistance from 2018 to 2019 is multiclonal. In this study, the ST 405 and ST 156 having mcr-1 are reported for the first time from Pakistan. The ST 405 and ST 156 have been previously reported in association with carbapenem-resistant b/aNDM-5 from clinical isolates and chicken meat, respectively [26 27]. In other regions of the world, the ST 405 and ST156 harboring mcr-1 have been reported, such as ST 405 in the United States and Algeria and ST 156 in China and Brazil [28-31]. ST 405 and ST156 belong to extraintestinal pathogenic $E$. coil, and avian pathogenic $E$. coli lineage, respectively. They are mainly involved in the global spread of blaCTXM-15 type extended-spectrum $\beta$ lactamases [28 30]. A recent study from Pakistan reported $m c r-1$ harboring ST117 in the broiler [32]. ST117 belongs to avian pathogenic E. coli lineage and may form a reservoir for human extraintestinal pathogenic $E$ coli and antimicrobial resistance[33]. The detection of $m c r-1$ in avian pathogenic and extraintestinal pathogenic $E$. coli in human clinical isolates is threatening because it supports the statement of $m c r-1$ transmission via the food chain [34].

All the four isolates have been successfully trans-conjugated to E. coli EC600. The S1 PFGE, southern blot, and PBRT results revealed that the $m c r-1$ gene was present on $60 \mathrm{~Kb}$ Incl2 plasmid. The Antibiotic susceptibility profiles of transconjugants showed that only the $m c r-1$ antibiotic-resistant gene was present on Incl2 plasmid. The similar incl2 plasmid harboring only $m c r-1$ antibiotic resistance gene had been reported in ST 155 isolated from a healthy broiler in Pakistan. The insertion sequence IsAp/1 of $m c r-1$ in ST155 incl2 was missing, while the remaining genetic context was similar to pHNSHP45 [9]. The PCR based mapping of $m c r-1$ genetic context in our isolates revealed that almost all amplified genes were similar to pHNSHP45, except the truncated tnpA gene, missing IsAp/1. Globally, plasmid analysis of various Incompatibility types has shown that IsAp/1 is usually absent in IncX4 type plasmid while in Incl2 type plasmid, it is either absent or present [6]. The missing of IsAp/1 might be due to the coevolution via the acquisition of $I s A p / 1$ for speedy relocation into other plasmids [35]. Alternatively, it might reinforce the constancy of $m c r-1$ on the plasmid [36] 
The missing of IsAp/1 strengthens our statement of having the same plasmid in ST155 (detected earlier from healthy broiler) and in our isolates [9]. This finding of $m c r-1$ on ST117 and similar plasmid profiles with a previously detected plasmid from a healthy broiler suggests that both horizontal transfer and transfer via food chain might be responsible for disseminating colistin resistance $m c r-1$ gene in Pakistan.

\section{Conclusion}

We reported the $m c r-1$ gene in human clinical isolates belonging to avian pathogenic $E$. coli lineage. The plasmid analysis and sequence typing indicate $m c r-1$ spread via the food chain and horizontal transfer. It might have severe consequences in the community. Our data stresses the need for mcr-1 prevention in humans, food chains, veterinary, and the environment by involving health care professionals, researchers, and government authorities to take concrete steps to prevent colistin resistance among the population.

\section{Abbreviations}

$b / a=$ Gene encoding $\beta$-lactamase

CLSI= Clinical and Laboratory Standards Institute

$\mathrm{CMH}=$ Combined Militry Hospital

CTX-M= Cefotaximase Munich, $\beta$-lactamase of ESBL-A type

HMC= Hayatabad Medical Complex

KTH= Khyber Teaching Hospital

LB= Luria-Bertani

$m c r=$ mobilized colistin resistant gene

MLST = Multi locus sequence type

PBRT $=$ PCR base replicon typing

PetN= Phosphoethanolamine

PFGE=Pulse Field Gel Electrophoresis

PIMS= Pakistan Institute of Medical Sciences

$\mathrm{RMI}=$ Rehman Medical Institute

ST= Sequence Type

TEM= Temoneira, $\beta$-lactamase of ESBL-A type.

\section{Declarations}

\section{Ethics approval and consent to participate:}

Not Applicable

\section{Consent for publication:}




\section{Availability of Data:}

The data supporting the findings of this study are included within the manuscript and its supporting information.

\section{Competing interests:}

The authors declare that they have no competing interests.

\section{Funding:}

This work was supported by grants from Natural Science Foundation of China (number 31771310 to Xingyuan Yang) and Anhui Province Natural Science Foundation (number 1708085MC67 to Xingyuan Yang). The funders had no rule in the study design, data collection and analysis, manuscript writing, proof reading and decision to publish.

\section{Author contributions:}

Study concept and design: HB and XY, Acquisition of data: HB. FH, and MAK, Analysis and interpretation of data: $\mathrm{HB}, \mathrm{ZGH}, \mathrm{JH}$ and $X Y$, Drafting of the manuscript: $\mathrm{HB}, \mathrm{XY}, \mathrm{ZGH}$ and $\mathrm{JH}$, Critical revision of the manuscript for important intellectual content: $\mathrm{XY}$, TR and MAK., Administrative, technical, material support and institutional study supervision: XY. All the authors read and approved the final version of the manuscript.

\section{Acknowledgement:}

The authors are thankful to the Institute of Basic Medical Sciences, Khyber Medical University Peshawar, Pakistan and Institute of Health sciences, Anhui University, China for supporting and facilitating this work.

\section{References}

1. Theuretzbacher U, Bush K. Critical analysis of antibacterial agents in clinical development. Nature reviews Microbiology 2020;18(5):286-98 doi: 10.1038/s41579-020-0340-0.

2. Stefaniuk EM, Tyski S. Colistin Resistance in Enterobacterales Strains - A Current View. Polish journal of microbiology 2019;68(4):417-27 doi: 10.33073/pjm-2019-055.

3. Zhai YJ, Sun HR, Luo XW, et al. CpxR regulates the colistin susceptibility of Salmonella Typhimurium by a multitarget mechanism. The Journal of antimicrobial chemotherapy 2020 doi: 10.1093/jac/dkaa233.

4. Kim S, Woo JH, Kim N, et al. Characterization Of Chromosome-Mediated Colistin Resistance In Escherichia coli Isolates From Livestock In Korea. Infection and drug resistance 2019;12:3291-99 doi: 10.2147/idr.s225383.

5. Liu YY, Wang Y, Walsh TR, et al. Emergence of plasmid-mediated colistin resistance mechanism MCR-1 in animals and human beings in China: a microbiological and molecular biological study. The Lancet Infectious diseases 2016;16(2):161-8 doi: 10.1016/s1473-3099(15)00424-7.

6. Nang SC, Li J, Velkov T. The rise and spread of mcr plasmid-mediated polymyxin resistance. Critical reviews in microbiology 2019;45(2):131-61 doi: 10.1080/1040841x.2018.1492902.

7. El-Sayed Ahmed MAE, Zhong LL, Shen C. Colistin and its role in the Era of antibiotic resistance: an extended review (20002019). Emerging Microbes \& Infections 2020;9(1):868-85 doi: 10.1080/22221751.2020.1754133.

8. Mohsin M, Azam M, Ur Rahman S, et al. Genomic background of a colistin-resistant and highly virulent MCR-1-positive Escherichia coli ST6395 from a broiler chicken in Pakistan. Pathogens and disease 2019;77(7) doi: 10.1093/femspd/ftz064. 
9. Lv J, Mohsin M, Lei S, et al. Discovery of a mcr-1-bearing plasmid in commensal colistin-resistant Escherichia coli from healthy broilers in Faisalabad, Pakistan. Virulence 2018;9(1):994-99 doi: 10.1080/21505594.2018.1462060.

10. Azam M, Ehsan I, Sajjad Ur R, et al. Detection of the colistin resistance gene mcr-1 in avian pathogenic Escherichia coli in Pakistan. Journal of global antimicrobial resistance 2017;11:152-53 doi: 10.1016/j.jgar.2017.10.012.

11. Mohsin M, Raza S, Roschanski N, et al. Description of the First Escherichia coli Clinical Isolate Harboring the Colistin Resistance Gene mcr-1 from the Indian Subcontinent. Antimicrobial agents and chemotherapy 2017;61(1) doi: 10.1128/aac.01945-16.

12. Mohsin M, Raza S, Roschanski N, et al. First description of plasmid-mediated colistin-resistant extended-spectrum $\beta$ lactamase-producing Escherichia coli in a wild migratory bird from Asia. International journal of antimicrobial agents 2016;48(4):463-4 doi: 10.1016/j.jjantimicag.2016.07.001.

13. Wayne P. Performance standards for antimicrobial susceptibility testing. 28th ed: Clinical and laboratory standards institute, 2018. http://iacld.ir/DL/public/CLSI-2018-M100-S28.pdf

14. Vingataramin L, Frost EH. A single protocol for extraction of gDNA from bacteria and yeast. BioTechniques 2015;58(3):120-5 doi: $10.2144 / 000114263$.

15. Control CfD, Prevention. Standard operating procedure for PulseNet PFGE of Escherichia coli 0157: H7, Escherichia coli non0157 (STEC), Salmonella serotypes, Shigella sonnei and Shigella flexneri. Centers for Disease Control and Prevention, Atlanta 2013. https://www.cdc.gov/pulsenet/pdf/ecoli-shigella-salmonella-pfge-protocol-508c.pdf

16. Shafiq M, Huang J, Ur Rahman S, et al. High incidence of multidrug-resistant Escherichia coli coharboring mcr-1 and bla (CTX-M-15) recovered from pigs. Infection and drug resistance 2019;12:2135-49 doi: 10.2147/idr.s209473.

17. Feliciello I, Chinali G. A modified alkaline lysis method for the preparation of highly purified plasmid DNA from Escherichia coli. Analytical biochemistry 1993;212(2):394-401 doi: 10.1006/abio.1993.1346.

18. Southern E. Southern blotting. Nature protocols 2006;1(2):518-25 doi: 10.1038/nprot.2006.73.

19. Hameed F, Khan MA, Muhammad H, et al. Plasmid-mediated mcr-1 gene in Acinetobacter baumannii and Pseudomonas aeruginosa: first report from Pakistan. Rev Soc Bras Med Trop 2019;52:e20190237 doi: 10.1590/0037-8682-0237-2019.

20. Bilal H, Hameed F, Khan MA, et al. Detection of mcr-1 Gene in Extended-Spectrum $\beta$-Lactamase-Producing Klebsiella pneumoniae From Human Urine Samples in Pakistan. Jundishapur J Microbiol 2020;13(4):e96646 doi: 10.5812/jjm.96646.

21. Berrazeg M, Hadjadj L, Ayad A, et al. First Detected Human Case in Algeria of mcr-1 Plasmid-Mediated Colistin Resistance in a 2011 Escherichia coli Isolate. Antimicrobial agents and chemotherapy 2016;60(11):6996-97 doi: 10.1128/aac.01117-16.

22. Doumith $\mathrm{M}$, Godbole G, Ashton P, et al. Detection of the plasmid-mediated mcr-1 gene conferring colistin resistance in human and food isolates of Salmonella enterica and Escherichia coli in England and Wales. The Journal of antimicrobial chemotherapy 2016;71(8):2300-5 doi: 10.1093/jac/dkw093.

23. Quan J, Li X, Chen Y, et al. Prevalence of mcr-1 in Escherichia coli and Klebsiella pneumoniae recovered from bloodstream infections in China: a multicentre longitudinal study. The Lancet Infectious diseases 2017;17(4):400-10 doi: 10.1016/s14733099(16)30528-x.

24. Rhouma M, Letellier A. Extended-spectrum $\beta$-lactamases, carbapenemases and the mcr-1 gene: is there a historical link? International journal of antimicrobial agents 2017;49(3):269-71 doi: 10.1016/j.jjantimicag.2016.11.026.

25. Jeannot K, Bolard A, Plésiat P. Resistance to polymyxins in Gram-negative organisms. International journal of antimicrobial agents 2017;49(5):526-35 doi: 10.1016/j.ijantimicag.2016.11.029.

26. Qamar MU, Walsh TR, Toleman MA, et al. Dissemination of genetically diverse NDM-1, -5, -7 producing-Gram-negative pathogens isolated from pediatric patients in Pakistan. Future microbiology 2019;14:691-704 doi: 10.2217/fmb-2019-0012.

27. Baloch Z, Lv L, Yi L, et al. Emergence of Almost Identical F36:A-:B32 Plasmids Carrying bla (NDM-5) and qepA in Escherichia coli from Both Pakistan and Canada. Infection and drug resistance 2019;12:3981-85 doi: 10.2147/idr.s236766.

28. Mediavilla JR, Patrawalla A, Chen L, et al. Colistin- and Carbapenem-Resistant Escherichia coli Harboring mcr-1 and blaNDM5, Causing a Complicated Urinary Tract Infection in a Patient from the United States. mBio 2016;7(4) doi: 10.1128/mBio.01191-16. 
29. Bachiri T, Lalaoui R, Bakour S, et al. First Report of the Plasmid-Mediated Colistin Resistance Gene mcr-1 in Escherichia coli ST405 Isolated from Wildlife in Bejaia, Algeria. Microbial drug resistance (Larchmont, NY) 2018;24(7):890-95 doi:

10.1089/mdr.2017.0026.

30. Yang RS, Feng Y, Lv XY, et al. Emergence of NDM-5- and MCR-1-Producing Escherichia coli Clones ST648 and ST156 from a Single Muscovy Duck (Cairina moschata). Antimicrobial agents and chemotherapy 2016;60(11):6899-902 doi: 10.1128/aac.01365-16.

31. Rossi F, Girardello R, Morais C, et al. Plasmid-mediated mcr-1 in carbapenem-susceptible Escherichia coli ST156 causing a blood infection: an unnoticeable spread of colistin resistance in Brazil? Clinics (Sao Paulo, Brazil) 2017;72(10):642-44 doi: 10.6061/clinics/2017(10)09.

32. Azam M, Mohsin M, Johnson TJ, et al. Genomic landscape of multi-drug resistant avian pathogenic Escherichia coli recovered from broilers. Veterinary microbiology 2020;247:108766 doi: 10.1016/j.vetmic.2020.108766.

33. Manges AR, Johnson JR. Food-borne origins of Escherichia coli causing extraintestinal infections. Clinical infectious diseases 2012;55(5):712-9 doi: 10.1093/cid/cis502.

34. Elbediwi M, Pan H, Jiang Z, et al. Genomic Characterization of mcr-1-carrying Salmonella enterica Serovar 4,[5],12:i:- ST 34 Clone Isolated From Pigs in China. Frontiers in bioengineering and biotechnology 2020;8:663 doi: 10.3389/fbioe.2020.00663.

35. Kuo SC, Huang WC, Wang HY, et al. Colistin resistance gene mcr-1 in Escherichia coli isolates from humans and retail meats, Taiwan. The Journal of antimicrobial chemotherapy 2016;71(8):2327-9 doi: 10.1093/jac/dkw122.

36. Snesrud E, He S, Chandler M, et al. A Model for Transposition of the Colistin Resistance Gene mcr-1 by ISApl1. Antimicrobial agents and chemotherapy 2016;60(11):6973-76 doi: 10.1128/aac.01457-16.

\section{Figures}




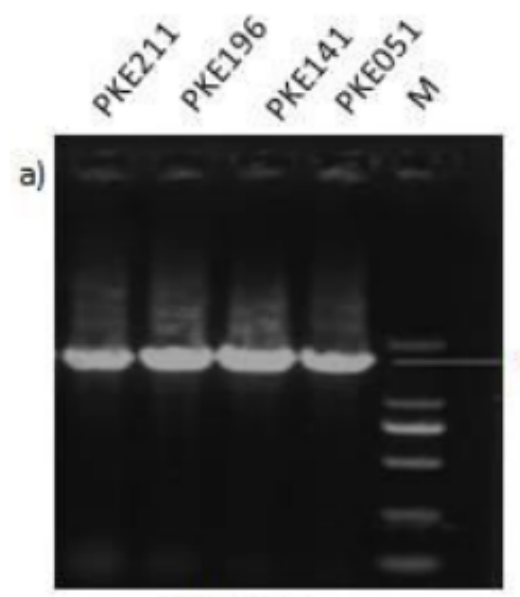

1554 bp

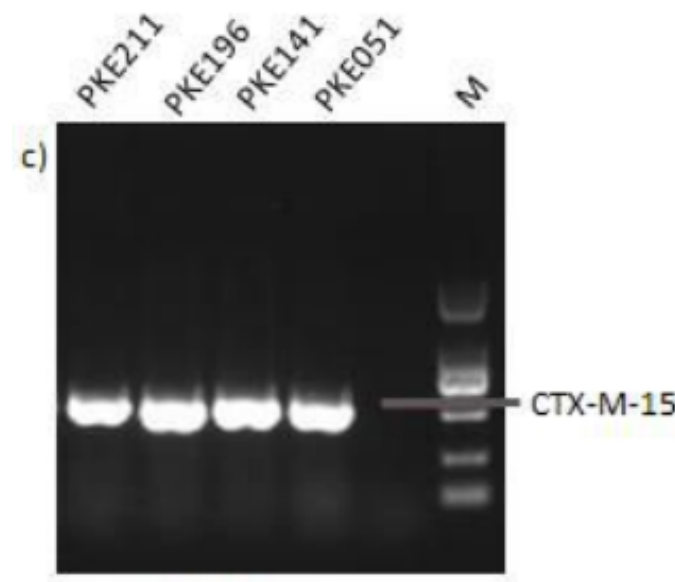

$550 \mathrm{bp}$

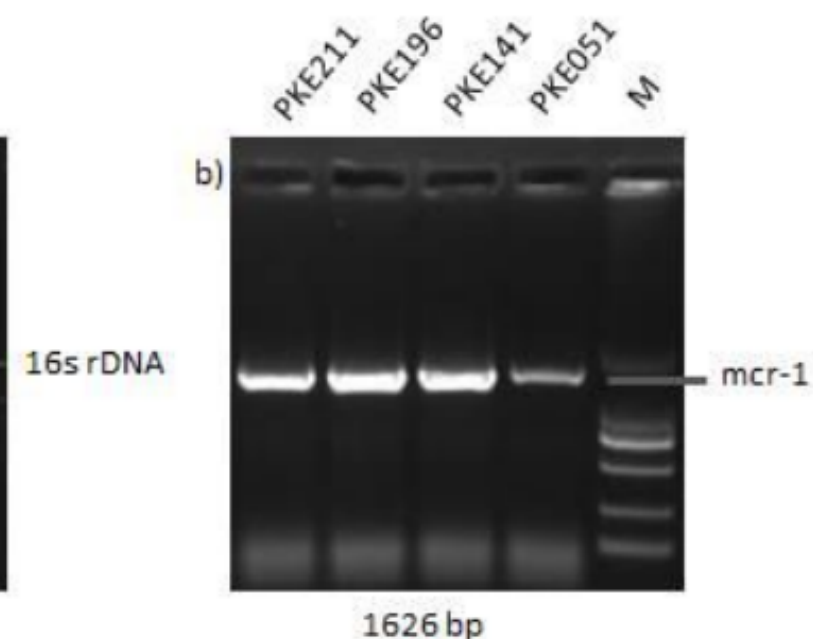

$1626 \mathrm{bp}$

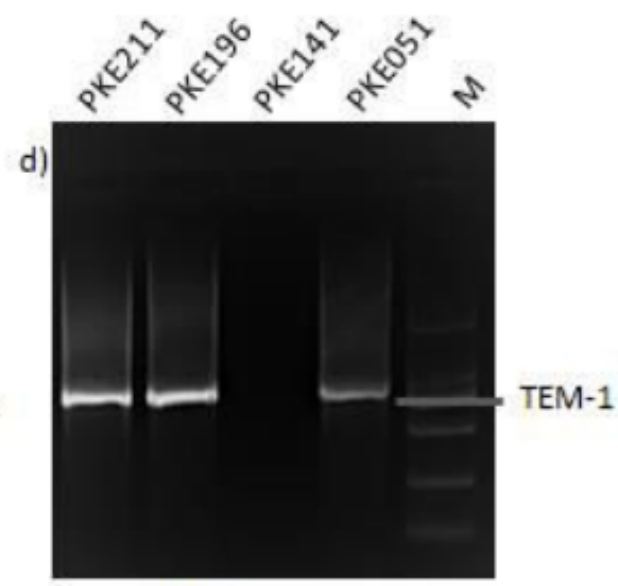

$800 \mathrm{bp}$

Figure 1

PCR results; a) 16s rDNA (1554bp), b) mcr-1 (1626bp), c) blaCTX-M-15 (550bp) and d) blaTEM-1 (800bp) on 1\% Agarose gel, PKE051, PKE141, PKE196 and PKE211 are Isolates ID and M is for 2000pb marker.

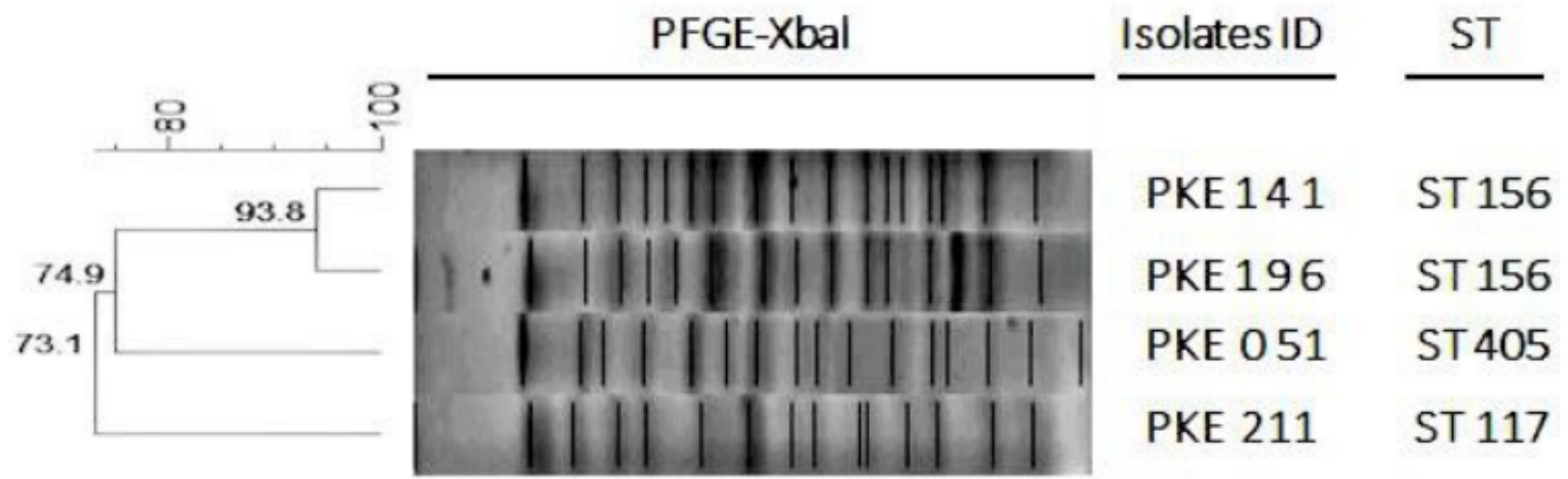

Figure 2

Xbal PFGE result of PKE141 (ST 156), PKE196 (ST 156), PKE051 (ST 405), PKE211 (ST 117), cluster analysis, and dendrogram is constructed by BioNumerics $\vee 8.0, \mathrm{ST}$ is abbreviated for sequence type. 


\section{M8 M7 M6 M5 M4 M3 M2 M1 M}

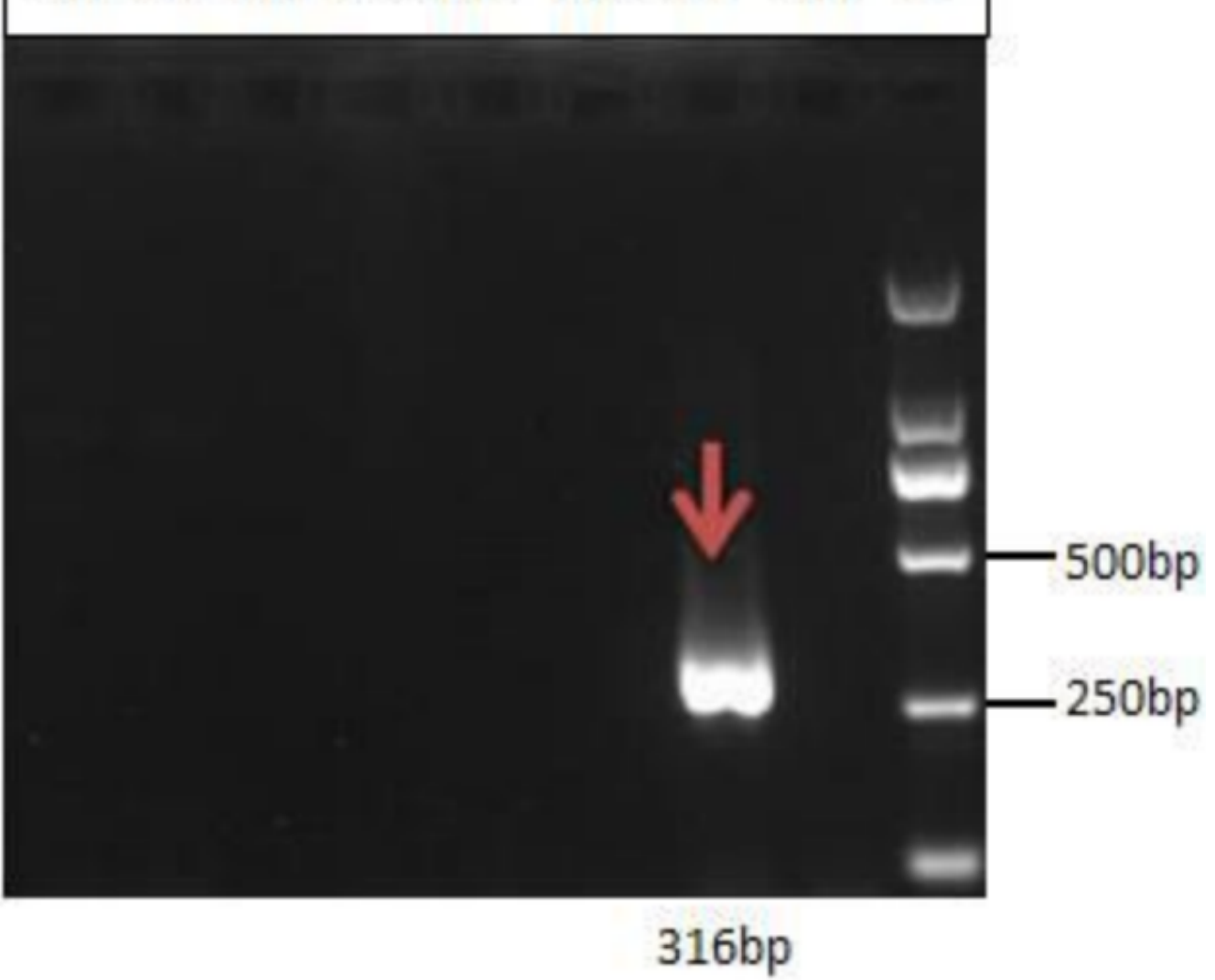

\section{Figure 3}

PBRT of transconjugants on 2\% Agarose gel. M1 to M8 are the eight multiplex PCR, M is 2000bp marker, only M2 shows a band size of $316 \mathrm{pb}$, indicating Incl2 type plasmid.

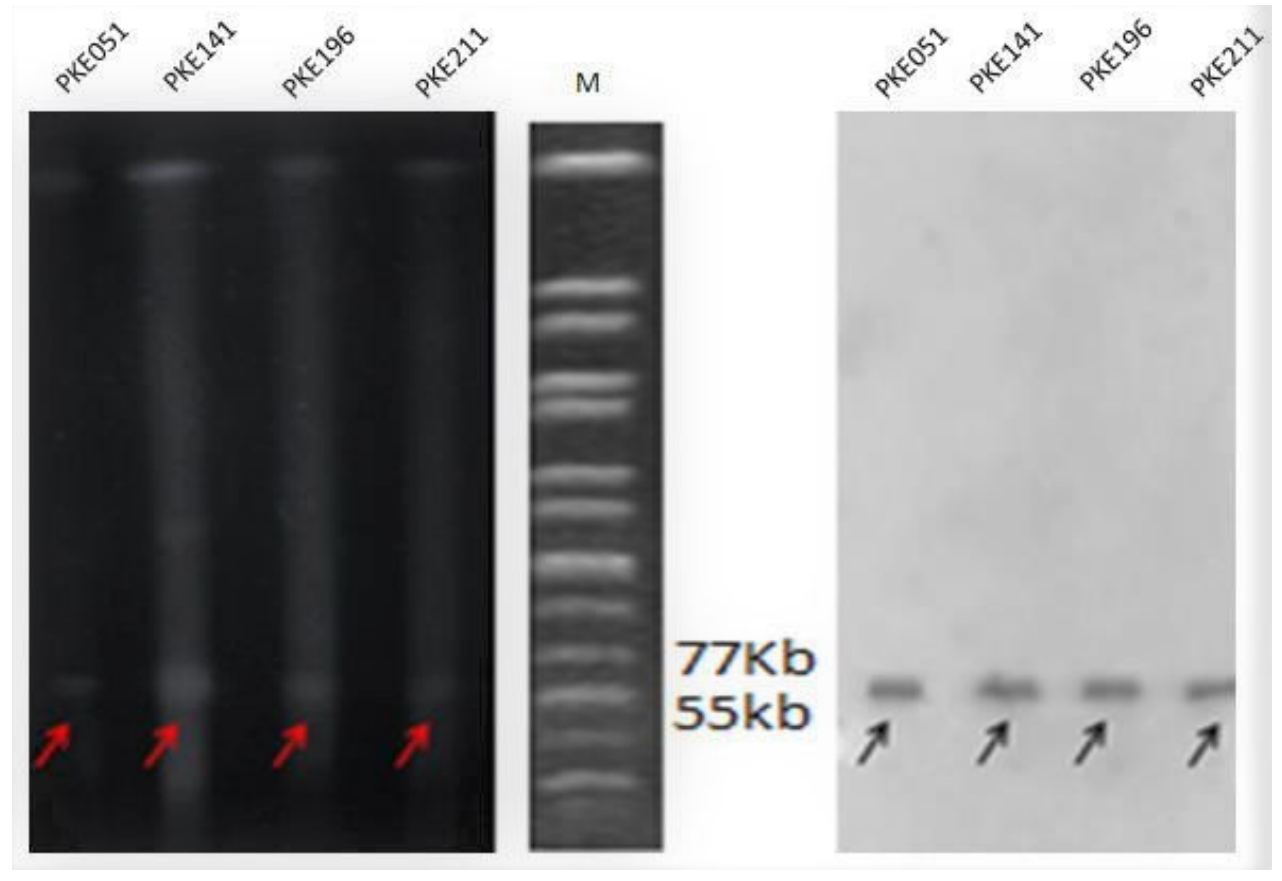

\section{Figure 4}

S1 PFGE and Southern blot, PKE051, PKE14, PKE196, and PKE211 are the isolates; M is the H9812 molecular size marker (1135 to 20.5kb) digested with Xbal enzymes. All strains show to 60kb plasmid on S1 PFGE gel and southern blot. 


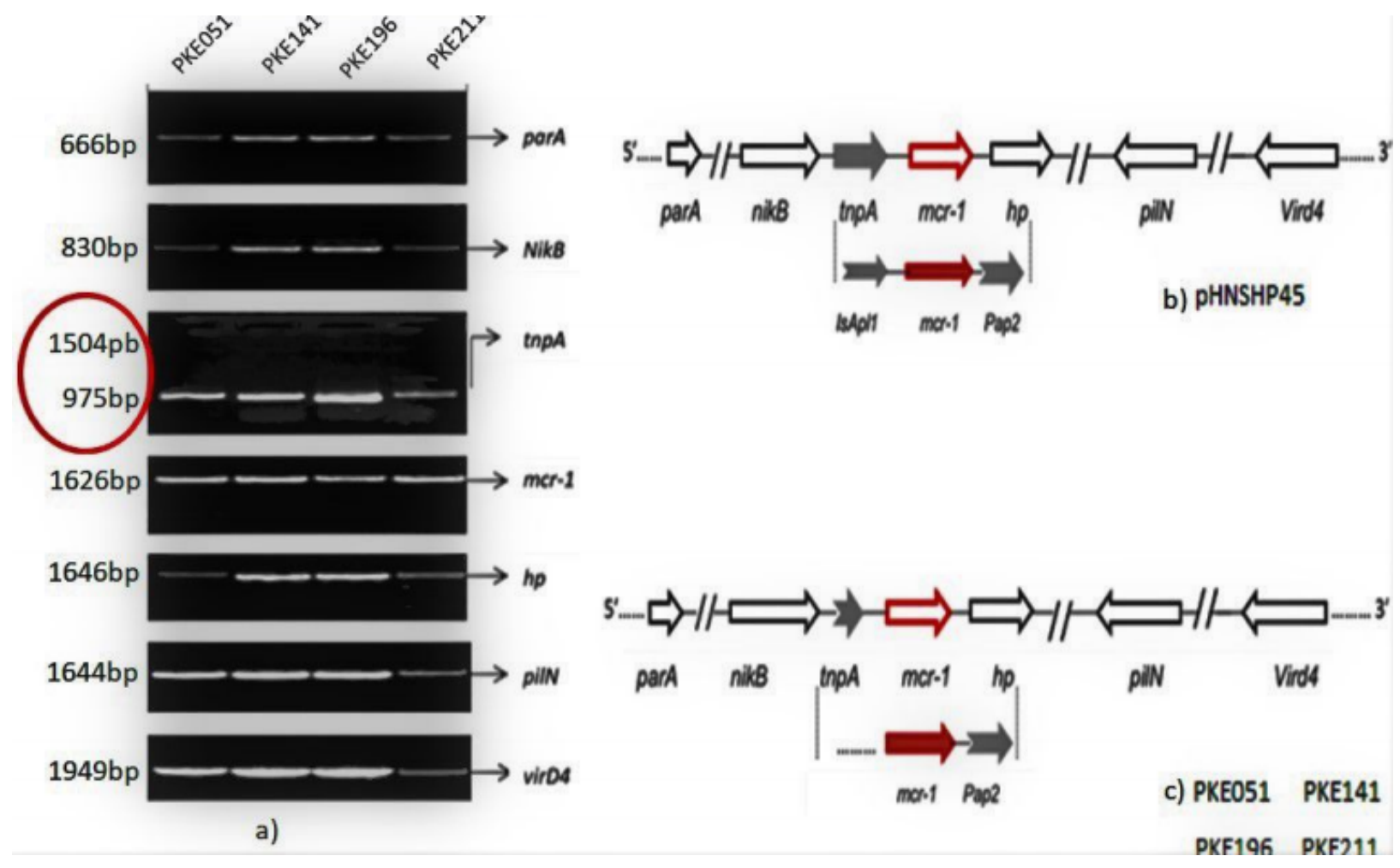

Figure 5

PCR based analysis of mcr-1 genetic context. a) PCR assay of six genes in the surrounding of mcr-1, red circle indicates the truncated band size of tnpA. b) Map of reference pHNSPH45 showing IsApl1 on upstream and Pap2 on downstream of mcr-1. c) Map of mcr-1 genetic context in our isolates showing missing of IsApl1 due to truncated tnpA.

\section{Supplementary Files}

This is a list of supplementary files associated with this preprint. Click to download.

- additionalfile.docx 